\title{
DEVELOPING A DISTRIBUTED MEETING SERVICE TO SUPPORT MOBILE MEETING PARTICIPANTS
}

\author{
Seamus Hickey MEng \\ Deptartment of Information Processing Science, University of Oulu, Finland
}

Key words: Distributed meetings, Mobile Services, Telepresence

Abstract: $\quad$ Future $3^{\text {rd }}+$ generation mobile wireless systems are been designed to support high data rates in comparison to existing voice based wireless systems. The success of these new systems will partly be based on the data services it can provide. Apart from supporting traditional data (email, ftp etc.) \& multimedia applications (videoconferencing, web access etc.), the combination of mobility and high speed data terminals will lead to innovative new data services e.g. navigation. In the PAULA project we are implementing an experimental distributed meeting service, based on Telepresence. The service attempts to compensate for the 'in transit' state of a mobile user by enabling them to attend a remote meeting while providing a high level of immersion, or sense of presence, at the meeting. The experimental system comprises of an omnidirectional camera placed in the meeting room that provides a constant navigable video stream to a remote mobile participant utlilising a Head Mounted Display.

\section{INTRODUCTION}

Current mobile phone systems have predominantly focused on voice services while providing only basic data rates up to $14.4 \mathrm{kbps}$. Considerable interest has now focused on providing higher mobile data rates to support basic data services (e.g. email, ftp) and multimedia applications. Towards this end the introduction of the GPRS data service in 1999 will provide data rates up to $170 \mathrm{kbps}$ [1]. In addition, research and standardisation of $3^{\text {rd }}$ 
generation mobile services (e.g. UMTS) is likely to facilitate data rates up to 2 Mbps for indoor systems and 384kbps for outdoor [2] by 2002. With the introduction of these new data rates, the mobile user should not only have access to most of the services available from a fixed computer or workstation, but also have these services anytime and anywhere. In essence, the mobile user has the additional service of mobility. The combination of high data rates and mobility can lead to innovative new services like navigation [3]. These new services can also provide support to a mobile user who would normally be disadvantaged while been in transit between destinations or physically located outside of the normal working environment. The service described in this paper relates to supporting such a user, who must attend a meeting at a remote venue and to develop the service sufficiently to compensate for the spatial disadvantage. In this paper we concentrate on the nature of this type of service and will use the knowledge obtained to determine the supporting network requirements.

Distributed meetings, both formal and informal, have attracted considerable research interest. Various different methods have been proposed with a wide range of technical sophistication [4]. The various systems include simple teleconference systems to full Collaborative Virtual Environments (CVE). Until recently, the point of access of these systems has been fixed, e.g. workstations or desktop computers, and very little attention has focused on support for mobile participant's [5]. In general the distributed meeting models under research consists of two types, those requiring all the participants to be distributed and convene in a virtual setting, or specialised meeting room environments with participants communicating over dedicated links. These models do not cater for a more normal situation where the majority of the meeting participants exist in a normal meeting room setting, but a handful of desired participants are remotely located. Traditionally the only manner in which these remote participants could attend was by phone. However, even with normal video conferencing, such methods are not entirely satisfactory as the remote participant is deprived of many normal interactions available by been physically present, preventing them from been as effective as if they were they present $[6,7]$.

To compensate and provide a higher sense of immersion to the mobile user, a distributed meeting service based on telepresence techniques is proposed. An experimental distributed environment has been implemented which helps to give a higher sense of immersion than is normally available from video conferencing systems. This paper describes the components of the system, provides other research that supports this work, describes the current experimental system, discusses the results and identifies future work and improvements to refine the service. 


\section{CURRENT DISTRIBUTED MEETING MODELS}

Current research models for distributed meetings have focused on 4 areas, media spaces $[8,9,10]$, spatial video conferencing $(11,12$.$) ,$ Collaborative Virtual Environments (CVE) [13,14,15], and telepresence $[16,4]$.

Media Spaces. Media Spaces enhance an existing workplace (e.g. an office) by using audio/video services as a basis for providing a range of general services. These services support social browsing, peripheral relationships and the establishment of long term relationships between physically separated people. Examples of such systems are RAVE [10] and Cruiser [7]. Experiences with the Cruiser system indicate that this medium was used primarily as a precursor to longer meetings in which all the participants were co present.

Spatial Video Conferencing. Spatial Video Conferencing covers video and audio communication over a desktop system or through dedicated links between public meeting rooms. These Video conferencing systems are sometimes enhanced with support for collaborative and shared work spaces. Video Conferencing systems between dedicated meeting rooms involve a significant support and logistics burden, which creates an obstacle to its adoption [12]. Furthermore, the lack of gaze direction, an important element in meeting environments, is a major disadvantage to such systems. Solutions to the gaze awareness problem have led to systems like MAJIC [11]. However MAJIC is limited to a maximum of 4 meeting participants.

Collaborative Virtual Environments (CVE). CVEs are networked virtual reality systems where each meeting participant is represented by an avatar, a virtual graphical embodiment of a real person, in a virtual reality meeting room. Within the virtual meeting room, the participants may engage in social and collaborative work. Experience with CVE's [14] has highlighted that extensive collaborative work is needed to first set up the meeting. Also, the embodiment does not accurately reflect the state of its 'real' meeting participant, e.g. the participant may have left the office or is performing another task, yet the embodiment still exists in the virtual meeting room. This can lead to confusion between the meeting participants.

Telepresence. Telepresence allows users to experience a remote physical space through computer and communication technologies. Typically, the person is represented in the remote space by a physical proxy e.g. robot. The user may experience a good level of immersion by using a Head Mounted Display (HMD), where live video is displayed instead of $3 \mathrm{~d}$ graphics. One of the most notable examples of such a system is the GestureCam [16]. 
From a review of the current research into distributed meetings, it would appear that the results from the use of a Telepresence system could provide the best means of communication support for a mobile user. In the design of such a service, it is important to highlight the major factors that should be considered in the design.

The GestureCam[16] experiment comprised of a remote user attending a meeting whereby he is attempting to instruct the meeting participants on how to operate a piece of equipment. A camera was mounted on an actuator and placed in the meeting room. The camera was controlled by the remote user, who instructed it to move in which ever direction he so wished. Some major conclusions from this work indicated that an additional camera was required to provide a wider angle of view. A similar problem was encountered with Media spaces [17]. In the MTV experiment, it was noted that there were 'significant constraints due to the limited field of view offered by a single fixed camera' and it concluded that 'techniques that allow continuos camera movement in remote spaces might overcome the limitations we observed in this study'. These conclusions argued well for the use of physical proxy.

There are a number of problems with using a physical proxy. Apart from the obvious problem of maintaining a mechanical device, in practice, only one person can have control of the proxy at any one time. Controlling who would have control of the view is problematic enough, yet it is certain that the view that is suitable for one person may not suit another.

An important factor to consider in the design of a telepresence system is the significant problems that can occur with the meeting setup. In general, the complexity or difficulty in establishing the meeting should not be such, that it would discourage people form using it.

In an attempt to counteract these problems an omni-directional camera, which has a Field of View (FOV) of 360 degrees, is used as a physical proxy. An omni-directional camera can provide a 360 degree FOV, while been fixed in one position. This removes the need for maintaining a mechanical device. In addition, with the use of supporting software the camera can support multi -user access.

\subsection{Distributed Meeting Service Architecture}

The distributed meeting architecture is shown in Figure 1 and is comprised of a number of components. The premise of the architecture is to support a mobile user who wishes to attend a meeting at a remote location. The components of the architecture are based on a mobile user, who is 
utilising an advanced mobile terminal with support for a HMD, service profiles sitting on top of a bearer service (e.g. WLAN), and a meeting room which contains a camera capturing device and bi-directional audio conferencing equipment. The mobile user and the meeting room terminal each has it's own service profile, with the meeting room terminals profile been controlled by the provider of the meeting room.

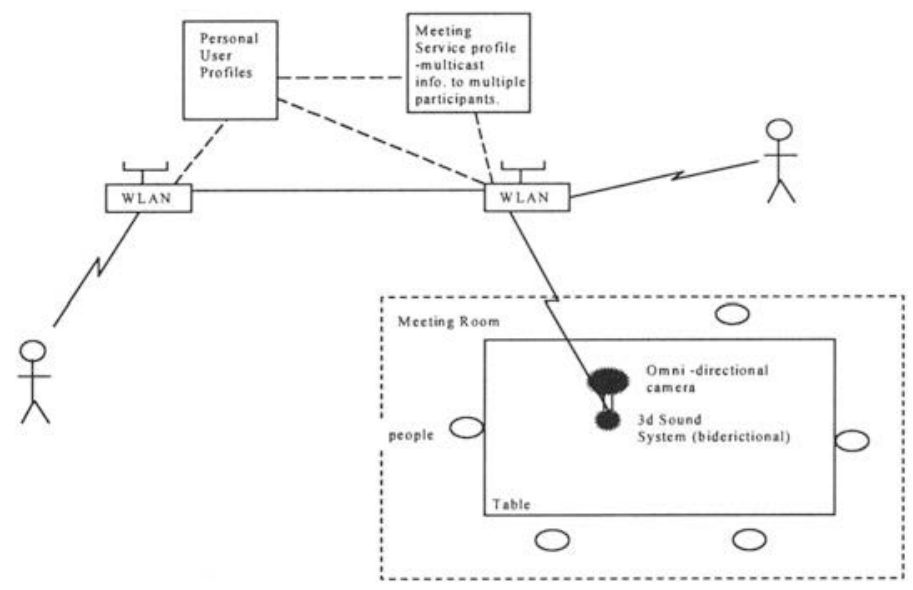

Figure 1. A Distributed meeting service architecture supporting multiple users

\subsection{Users Mobile Terminal}

The mobile data terminal required to support the distributed meeting service described so far, requires an advanced data terminal capable not only of providing high data rate access, but has a sophisticated display technology, such as support for a HMD. While no such terminal currently exists commercially, considerable research effort is underway into realising this technology. The CyPhone [18] is one of the first instantiations of such a terminal and is shown in figure 2(a). A future iteration of the terminal is shown in figure 2(b). It is conceivable that such terminals can support Telepresence and Augmented Reality services. It is also anticipated that in the next decade or so, that HMD's may be as widely available as Walkman's are today [19]. The popularity of such devices may result from people preferring to use these devices instead of a more cumbersome laptop computer and indeed observations of people at meetings showed them using Personal Digital Assistants (PDA) in preference to laptops [20]. 


\subsection{Service architecture}

In line with the current trend in mobile communications, the mobile user will have their own personnel service profile. The service profile will have features that indicate its ability to use advanced visual services like the distributed meeting service. In our current setup, the user service profile is trivial. The meeting room terminal's service profile may contain considerably more service features. Not only would it control user access to the meeting, but it could provide additional services such as recording minute meetings, storing data, scheduling etc. The bearer service used is a 2 Mbps WLAN. This was chosen to mirror the anticipated bandwidth that will be available from UMTS.

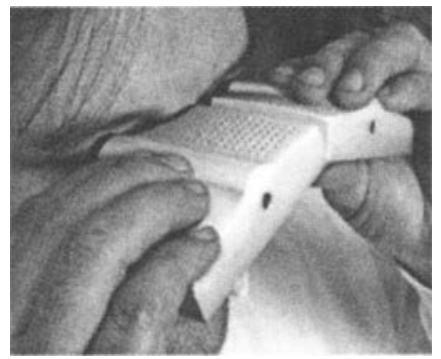

(a)

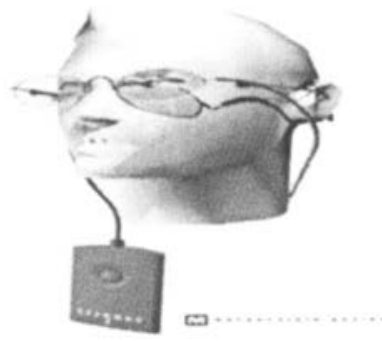

(b)

Figure 2(a). An example of a CyPhone with stereoscopic display.

2(b). A proposed future version of the CyPhone with support for a Head Mounted Display.

\subsection{Meeting room terminal}

The data terminal that occupies the meeting room contains an image capturing devices and bi-directional audio conference equipment. The preferred image capturing device is an omni-directional camera that is capable of taking a 360 degree video stream of the surrounding environment.

\section{DISTRIBUTED MEETING TEST ENVIRONMENT.}

An experimental distributed meeting service has been established at the University of Oulu. The experimental system is based on the model shown in figure 1, with a number of differences. Firstly, the project does not use a 
sophisticated data terminal like the CyPhone. Also, the service structure has not been implemented entirely, only the WLAN part has been used in the experiment.

The meeting room setup consists of the CycloVision ParaCamera, which is connected to a Matrox Meteor II frame grabber that continuously grabs a constant stream of video images. The CycloVision ParaCamera shown in figure 3, is comprised of the Panasonic colour digital camera GP-KR222 and an optical system comprising of special lens and a parabolic mirror that captures a 360-degree view of the surrounding area. The Field Of View (FOV) of the ParaCamera is 360 degrees horizontally and 180 degrees vertically. The captured image is called an 'obstructed image', as the image is partially obstructed by the camera itself and the metal holding the mirror. Each recorded frame is called a ParaFrame and shows the entire 360 degree view in one frame. This is difficult to interpret by a normal human user. Additional software is required to convert the ParaFrame into a normal 'perspective' view. This perspective view provides a narrower FOV and the video image is that which would be expected from a normal video camera. Additionally, it is possible to dynamically change the perspective using a mouse or head tracking system to navigate the ParaFrame. CycloVision provides ParaPlayer software to convert the 360-degree video stream into a perspective frame. However, this software was not suitable for our purposes because it did not support a full screen mode, which is essential to provide a sense of immersion using the Head Mounted Display. Therefore new proprietary software was developed to perform the perspective transforms in full screen mode. One side affect of converting the images to a full screen mode, is that it has a degrading affect on the image resolution.

The ParaCamera was placed on the corner of the table. Originally it had been intended to invert the camera and suspend it from the ceiling, i.e. with the parabolic lens towards the ceiling. However, finding the optimal height to suspend the camera was problematic. This optimal position for viewing participants sitting down had the side affect of 'cutting off' the upper body of a standing participant. Manually changing the camera position to compensate was not considered as an option because it would require excessive fidgeting which would prove an annoying diversion to achieving the meetings objective. Consequently the camera was placed with the parabolic lens lying on the table, as shown in figure 4 . This solution was not entirely satisfactory, as the video image suffered due to interference from light sources e.g. light bulbs, sunlight. The camera can be modified to compensate for excessive light intensity.

The ParaCamera shown in figure 4 is the spatial representation of the remote mobile user in the meeting room. Initially it had been intended to place it in the centre of the room, but to provide a more natural feeling, it 
now approximates the position a person would take were they to physically attend the meeting

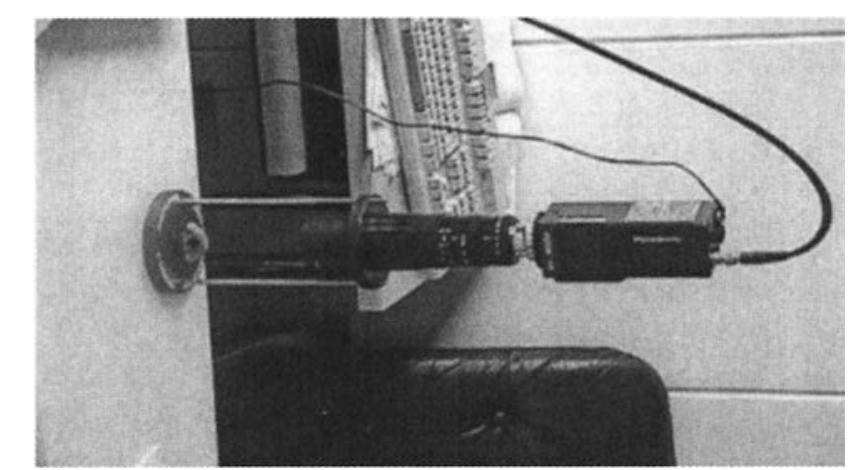

Figure 3. A CycloVision ParaCamera, consisting of a colour camera, specialised optics and a parabolic mirror.

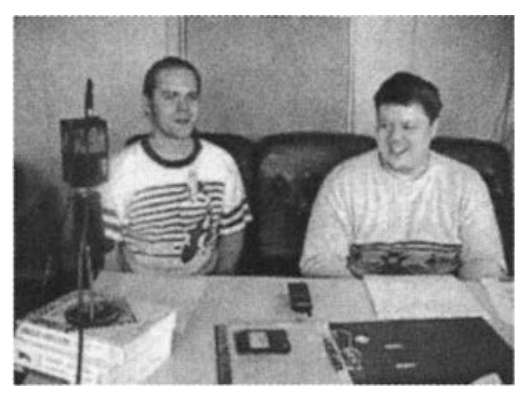

Figure 4. The meeting room with two participants. The ParaCamera is placed on some books

The mobile user was located in a second room on the same floor, but approximately 20 meters away. The mobile user wore a Head Mounted Display. The Head Mounted Display, shown in figure 5, was attached to a computer and connected to a Polhemus tracking system. The Polhemus tracking system is required to monitor the exact head position of the remote user. The head position co-ordinates are transmitted to the computer where they are used to calculate the perspective view for the mobile user. The perspective view is then displayed on the HMD. By moving his head the remote user can browse the remote meeting room. 


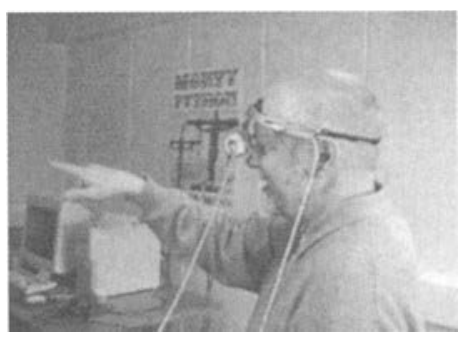

Figure 5. The mobile participant with a Head Mounted Display.

The meeting room video stream is transmitted to the remote user's computer over a WLAN. It is important to mention that the omni directional video stream is transmitted over the WLAN and at the remote user's end, the perspective image is calculated based on their head.

The environment was tested during one project meeting. The meeting consisted of six people, with one person as the remote participant. While initially there was considerable interest in the technology, the meeting managed to progress normally, despite a number of intrusions by curious onlookers. This affected the remote user more as people were unaware that he was attending a meeting.

\section{DISCUSSION}

The initial feedback from the trials has been encouraging. The ability of the remote user to look in whichever direction they desired was a strong point of the system. Users commented that they felt a higher sense of immersion than they had expected. The added advantage of getting visual feedback, or cues, from the other meeting participants in the remote meeting, was considered to be an improvement over audio only based communication, results are in line with other research work [7].

Observations of the remote user showed them changing their head positions in order to look around the room or focus on an individual in the remote meeting. The use of a HMD with live video feed was considered to be more 'immersive' than viewing the same video stream on a computer monitor.

The system as it presently exists has a number of both technical and perceptional problems, partially caused by the software, the camera system chosen and some general weaknesses in the system.

Implementing our own perspective transforms to provide a full screen mode for the HMD was achieved at the expense of resolution. The resolution 
was sufficiently poor so that the remote user had difficulties in recognising people beyond a distance of approximately 2 meters. While this was adequate for our meeting setup, which was based on a small meeting group, it is not adequate for larger meetings, or capable of supporting presentations. In our case, not many users seemed to be unduly concerned about the picture quality, as it was purely a discussion based meeting.

The main user comments centred on the frame rate, which was very slow, especially when users changed their head position quickly. It usually took a few seconds for the correct perspective view to correspond with the users head position. The video images were transmitted without compression and while we have developed compression algorithms, these were not working with the software at the time of these tests. However, the integration of the compression algorithms should improve the frame rate.

The affect of light sources on the camera considerably degraded the image at times. The mobile users complained about turning to see a person to their right, who was sitting by a window, and of being unable to see them properly because of the sunlight through the window. Consequently, it was necessary to draw the blinds in the room to improve the picture quality. While the camera was set for the average light intensity, there seemed to be 'light spots' which had a higher intensity of light in comparison to the normal light intensity.

Apart from the technical problems, a number of communication and perceptual problems occurred. One user complained that the original position of the camera, lying on the table, made him feel as though he was 'looking up' at everybody else. He asked us to raise the camera to his perception of eye level by placing some books under the camera. Additionally, a problem occurred when the mobile user was attempting to decide who was currently speaking. In a discussion afterwards, the mobile users commented that it was easier to know who was talking depending on their ability to recognise different voices. Those unable to recognise a meeting participant's voice had a harder time discerning that speaker. It was noted that the slow frame rate and poor resolution did not help pick out who was talking, but observing the direction in which the other meeting participants were looking did help.

From the viewpoint of those physically attending the meeting, it was noticed that the introduction of the system had little affect on their normal behaviour. These participants were largely acting as though it was a normal phone conference, as they only had an audio connection. These meeting participants had a number of observed problems with the audio system. On a number of cases they were forced to ask if the remote participant was still there as there was no feedback from him. On one occasion, the mobile user was showing the HMD to another person who had just walked in to the 
room, which was unknown to the people in the meeting room who were trying to get his attention. This is a general problem with audio only communication and also recorded by those working with CVE's.

On another occasion, the mobile user attempted to verbally communicate information (an email address) to the meeting room participants. To clarify the information, the meeting participants wrote out the given email address on a large sheet of paper that they showed to the camera in order to confirm that it was correct. It can be concluded that the efficient transfer or sharing of information is a considerable weakness in the system that needs to be addressed.

While the meeting participants generally treated the remote mobile user as a normal phone user, it was observed that they often turned to the camera to address the user. Of course this phenomena could also be the result of addressing the audio connection which was co-located with the video camera. The affect of doing this was quite natural to the users who seemed to relate the camera to the 'presence' of the remote mobile user.

\section{IMPROVEMENTS AND FUTURE WORK.}

The experimental distributed meeting environment described in this paper has given us initial feedback and results which highlight both the advantages and problems in this service concept. The feasibility of the service depends on 2 criteria, the anticipated wide availability of cheap HMD, and adding sufficient features to the service so as to encourage mobile users to select it as the preferred method of communication [21]. With this in mind, we are moving to firstly improve on the system by taking the experimental results into consideration and solving the immediate problems.

The distributed meeting environment described in this paper requires some modifications to improve its performance and usability. The most immediate work will concentrate on implementing the compression algorithms to improve the frame rate. This was the primary issue raised by those who tried the system.

The main computing complexity resides at the remote user's location, as this is where the perspective transforms are calculated. In real terms this would mean that the mobile terminal would require significant computing power, increasing the expense of the terminal. Alternatively, the perspective image could be calculated in the network backbone. In this scenario, the mobile terminal will send the head position co-ordinates to the backbone which computes the perspective image. A comparison of the two techniques in terms of benefits and efficiency would be interesting. 
In addition, the current environment only supports a single user. Expanding the system to support multiple mobile users should give us some idea of the problems they face. The implementation of a protocol to manage multiple user support is under development.

To solve the problem of the poor video resolution will require a more powerful omni-directional system. An 'Image Based Rendering' system using an omni-directional camera comprising of a matrix of 8 cameras and morphing software has been developed at the University of Tokyo [22]. This system offers a significant improvement on resolution but does not perform in real-time as yet. However, the approach taken provides a possible solution to the poor resolution offered by the ParaCamera.

As the audio connection is also of major importance, a 3D sound system will be added to assist in the location of a speakers voice and to provide a deeper sense of immersion. To provide some form of visual user feedback to the meeting room participants, we will explore the use of 'talking heads' displayed on a monitor at the location of the omni-directional camera. Another solution could involve having a miniature camera as part of the mobile user's data terminal, as suggested in the CyPhone project, providing a video stream to the meeting room participants.

Improving collaborative work and the transfer of data will require innovative new techniques. Most of the existing collaborative techniques are not suitable for mobile users unless they have a laptop, or some access to a desktop computer. One innovative new method that will be explored, as a possible solution, is to incorporate 'augmented reality' objects in the meeting room [23]. Augmented reality objects are computer graphical objects that can be superimposed upon the video stream. A user can interact with these objects to perform functions or tasks. These objects can represent various items in the meeting room, which can be selected by the mobile user and enable him/her to send and receive information e.g. slides, emails etc.

\section{CONCLUSIONS}

A distributed meeting service supporting mobile users has been introduced. A possible service architecture has been described based on Telepresence and the justification for this work and approach taken has been provided. The advent of high mobile data rates and sophisticated data terminals supporting Telepresence and Augmented Reality techniques supports the feasibility of this service.

An experimental telepresence system using a CycloVision omnidirectional ParaCamera and a remote user connected to a HMD have been demonstrated. The system has been shown to provide a more flexible ability 
to view the surrounding area of a remote meeting and is capable of supporting multi-user access. Results have been encouraging and the plausibility of the system has been sufficiently justified to merit further research and expenditure of resources.

Furthermore, areas of improvement have been identified. Of priority will be improving the frame-rate by adding compression algorithms, an efficient broadcast protocol to provide multi-user access, a 3D sound system to provide a deeper sense of immersion, support for $3 \mathrm{D}$ talking heads and incorporating augmented reality objects to enable user interaction with the environment.

\subsection{Acknowledgements}

This project is sponsored by the PAULA project. The PAULA project is supported by the Academy of Finland and is a co-operative work between the University of Oulu, Helsinki University of Technology, VTT Oulu and Tampere University of Technology. I would also like to thank Tino Pyssysalo, Prof. Petri Pulli, Sami Ahola and Prof. Kari Kuutti for their invaluable assistance during this project.

\section{REFERENCES}

1. Concepts, Services and Protocols of the New GSM Phase 2+ General packet radio Service. G. Brasche, B. Walke. IEEE Communications magazine, August 1997, Vol 35. No. 8. IEEE Communications Society

2. Research Activities on UMTS Radio Interface, Network Architecture and Planning. E. Berruto, M. Gudmundson, R. Menolascino, W. Mohr, (1998). IEEE Communications Magazine, Feb. 1998, Vol. 36 No. 2

3. Services and Applications: Requirements and realisations in the UMTS Era. A. Munro, E. Geulen, M. Deguine,G. Melpignano. 1998 IEEE Communications Magazine, Feb. 1998, Vol. 36 No. 2

4. Shared Spaces: Transportation, Artificiality and spatiality. S. Benford, C. Brown, G.

Reynard, C. Greenhalgh. Proc. Of ACM 1996 Conference on CSCW. Boston, Massachusetts.

5. Mobility in Collaboration. P. Luff, C. Heath. Proceedings of ACM 1998 conference on CSCW. ACM Press.

6. A comparison of face to face and distributed presentations. E. Isaacs, T. Morris, T Rodriguez. Human Factors in Computing Systems, CHI '95 Conference proceedings. ACM Press.

7. Evaluating video as a technology for Informal Communication. R. Fish, R. Kraut, R. Root. Human Factors in Computing Systems, CHI ' 92 Conference proceedings. ACM Press.

8. A Virtual Window on Media Space. W. Graver, G Smets, K. Overbeeke. Human Factors in Computing Systems, CHI '95 Conference proceedings 
9. Portholes: supporting awareness in a distributed Work group. P. Douish, S. Bly. Human Factors in Computing Systems, CHI '92 Conference proceedings. ACM Press.

10. Realising a video environment: Europarcs RAVE system. W. Gaver, T. Moran, A. MacLean. Human Factors in Computing Systems, CHI '92 Conference proceedings. ACM Press.

11. Multiparty Videoconferencing at Virtual social distance: MAJIC design. K. Okada, F. Maeda, Y. Ichikawaa, Y. Matsushita. ACM 1994 Conference on CSCW, Chapel Hill, North Carolina. ACM Press.

12. Automated Assistance for the telemeeting Lifecycle. N. Bergmann, J. Craig Mudge. ACM 1994 Conference on CSCW. Chapel Hill, North Carolina. ACM Press.

13. A Distributed Virtual Environment for concurrent Engineering. J. Maxfield, T. Fernando, P. Dew. Virtual Reality - Annual International Symposium 95, IEEE Computer Society Press.

14. Practically accomplishing Immersion: Cooperation in and for Virtual Environments. J. Bowers, J. O'Brien, J. Pycock. Proc. Of ACM 1996 Conference on CSCW, Boston, Massachusetts, ACM Press.

15. MASSIVE: A Collaborative Virtual Environment for Teleconferencing. C. Greenhalgh, S. Benford. ACM Transactions on Computer-Human Interaction, Vol. 2, No. 3, Sept. 1995.

16. GestureCam: A video communication system for sympathetic remote collaboration. $\mathrm{H}$. Kuzuoka, T. Kosuge, M. Tanaka. ACM 1994 Conference on CSCW. ACM Press.

17. One is not enough: Multiple Views in a media space. W. Garver, A. Sellen, C. Heath, P.Luff. Human Factors in Computing Systems, INTERCHI '93 Conference proceedings. ACM Press.

18. CyPhone - Future Personal Telecooperation Device. P. Pulli, T. Pyssysalo, J. Similä, J. Metsävainio. Telecooperation. Proceedings of the XV.IFIP World Computer Congress, 31/8 - 4/9 1998,Vienna \& Budapest.

19. An Application of Augmented Reality in Future Mobile Communication services. T. Pyssysalo. Personal Wireless Communications.

20. Collaboration using multiple PDAs connected to a PC. B.A: Myers, H. Stiel, R. Gargiulo. Proceedings of ACM 1998 conference on CSCW- edited by S. Poltrock and J. Grundin. ACM Press

21. Beyond being There. J. Hollan, S. Stornetta, Human Factors in Computing Systems, CHI ' 92 Conference proceedings. ACM Press.

22. Building a Virtual World from a Real world. M. Hirose, T. Tanikawa, T. Endo. Mixed Reality. Merging Real and Virtual Worlds, Edited by Y. Ohta, H. Tamura.

23. A taxonomy of Real and Virtual World Display Integration. P. Milgram, H. Colquhoun. Mixed Reality. Merging Real and Virtual Worlds, Edited by Y. Ohta, H. Tamura. 